\title{
String monopoles, string walls, vortex skyrmions, and nexus objects in the polar distorted $B$ phase of ${ }^{3} \mathrm{He}$
}

\author{
G. E. Volovik ${ }^{1,2, *}$ and K. Zhang $\oplus^{1,3, \dagger}$ \\ ${ }^{1}$ Low Temperature Laboratory, Aalto University, P.O. Box 15100, FI-00076 Aalto, Finland \\ ${ }^{2}$ Landau Institute for Theoretical Physics, Acad. Semyonov Av., 1a, 142432, Chernogolovka, Russia \\ ${ }^{3}$ University of Helsinki, Department of Mathematics and Statistics, P.O. Box 68 FI-00014, Helsinki, Finland
}

(Received 28 March 2020; revised manuscript received 26 April 2020; accepted 28 April 2020; published 2 June 2020)

\begin{abstract}
The composite cosmological objects-Kibble-Lazarides-Shafi (KLS) walls bounded by strings and cosmic strings terminated by Nambu monopoles - could be produced during the phase transitions in the early universe. Recent experiments in superfluid ${ }^{3} \mathrm{He}$ reproduced the formation of the KLS domain walls, which opened the new arena for the detailed study of those objects in a human controlled system with different characteristic lengths. These composite defects are formed by two successive symmetry breaking phase transitions. In the first transition the strings are formed, then in the second transition the string becomes the termination line of the KLS wall. In the same manner, in the first transition monopoles are formed, and then in the second transition these monopoles become the termination points of strings. Here we show that in the vicinity of the second transition the composite defects can be described by relative homotopy groups. This is because there are two well-separated length scales involved, which give rise to two different classes of the degenerate vacuum states, $R_{1}$ and $R_{2}$, and the composite objects correspond to the nontrivial elements of the group $\pi_{n}\left(R_{1}, R_{2}\right)$. We discuss this on example of the so-called polar distorted $B$ phase, which is formed in the two-step phase transition in liquid ${ }^{3} \mathrm{He}$ distorted by aerogel. In this system the string monopoles terminate spin vortices with an even winding number, while KLS string walls terminate on half-quantum vortices. In the presence of magnetic field, vortex skyrmions are formed, and the string monopole transforms to the nexus. We also discuss the integer-valued topological invariants of those objects. Our consideration can be applied to the composite defects in other condensed matter and cosmological systems.
\end{abstract}

DOI: 10.1103/PhysRevResearch.2.023263

\section{INTRODUCTION}

The combined topological objects, such as strings terminated by Nambu monopoles [1] and Kibble-Lazarides-Shafi (KLS) walls bounded by strings [2,3], typically appear at two successive symmetry breaking phase transitions [4]. An example is provided by the axion solution to the strong CP problem [5-8], where two different phase transitions occur as the universe cools down. In the first one the cosmic strings are formed. Then when the cosmic temperature reaches the quantum chromodynamics (QCD) scale, domain walls are formed, while cosmic strings become attached to these walls.

Similar formation of the combined objects in successive phase transitions has been observed in the nanoconfined superfluid ${ }^{3} \mathrm{He}$ (in the so-called nafen) [9]. The confined geometry provides variety of new phenomena in this spin-triplet

\footnotetext{
*grigori.volovik@aalto.fi

${ }^{\dagger}$ kuang.zhang@aalto.fi
}

Published by the American Physical Society under the terms of the Creative Commons Attribution 4.0 International license. Further distribution of this work must maintain attribution to the author(s) and the published article's title, journal citation, and DOI. $p$-wave superfluid. Some new phases, which can never be stable in bulk ${ }^{3} \mathrm{He}$, are stabilized by the nanoconfinement. Among them the polar phase has been predicted $[10,11]$ and experimentally identified [12]. Later it became clear that the main reason of the dominations of the polar phase in nafen is the extension of the Anderson theorem [13] to the polar phase with columnar impurities: The transition temperature to the polar phase is practically not suppressed by the strands of nafen [14-16], as distinct from the other superfluid phases. Similar extension of the Anderson theorem was also discussed in connection with multiorbital superconductors [17]. Another signature of the Anderson theorem is the observation that the Dirac nodal line in the spectrum of Bogoliubov quasiparticles in the polar phase is not suppressed by nafen strands [18], giving rise to the detected $T^{3}$ dependence of the gap amplitude.

In the polar phase the half-quantum vortices (HQVs) have been observed [19]. Originally the HQVs were predicted to exist in the chiral superfluid ${ }^{3} \mathrm{He}-\mathrm{A}[20,21]$, but for 40 years they escaped observation because in this phase they are energetically unfavorable compared to the singly quantized vortices. Now the HQVs are easily created in ${ }^{3} \mathrm{He}-\mathrm{A}$ : The HQVs are first formed in the polar phase, where they are energetically favorable, and then they survive after the phase transition from polar phase to the A phase due to strong 
pinning [9]. The structure, spin dynamics, and spin polarization of HQV in the A phase were theoretically studied during the last few decades [22-24]. Both the polar phase and ${ }^{3} \mathrm{He}-\mathrm{A}$ are superfluids with the so-called equal spin pairing [25]. In such systems the HQV can be considered as the vortex in one spin component, and in the chiral superfluid ${ }^{3} \mathrm{He}-\mathrm{A}$ such vortex contains a single (isolated) Majorana zero mode in its core [26-28].

Both in the bulk $B$ phase and in the polar distorted $B$ phase in nafen, the HQVs are not supported by topology. However, earlier it was shown that the nonaxisymmetric core of the singly quantized vortex [29,30], which was observed in bulk superfluid ${ }^{3} \mathrm{He}-\mathrm{B}$ [31], can be considered as a pair of HQVs connected by the KLS wall $[32,33]$. This wall is rather short: only of few coherence length size. The similar wall between HQVs appears in the polar distorted $B$ phase in nafen after transition from the polar phase. But due to strong vortex pinning in nafen, the KLS wall bounded by pinned vortices does not shrink and keeps its macroscopic size. This allows experimental detection and identification of such composite objects [9].

Strictly speaking, the wall bounded by strings is not a topological object, since after the second transition the topological charge of the string does not exist anymore. In the same way the string terminated by monopole is not topological. Here we show that under certain conditions these combined objects become topological, being described by relative homotopy groups. Originally the classification in terms of the relative homotopy groups has been used if there is the hierarchy of the energy scale or length scales in the physical system [34-36], when each energy scale has its own well-defined vacuum manifold $R_{i}$-the space of the degenerate states. Here are some examples.

(i) The spin-orbit interaction in superfluid ${ }^{3} \mathrm{He}$ is small, and we have the order parameter vacuum manifold $R_{1}$ at short distances where the spin-orbit interaction can be neglected, and the submanifold $R_{2} \subset R_{1}$ at large distances, where the space of the order parameter is restricted by spin-orbit interaction [34]. The relative homotopy groups $\pi_{n}\left(R_{1}, R_{2}\right)$ give different types of the topologically stable combined objects. The $\pi_{1}\left(R_{1}, R_{2}\right)$ describes the planar topological solitons terminated by strings. Examples are combined spin-mass vortices with soliton tail observed in superfluid ${ }^{3} \mathrm{He}-\mathrm{B}$ [37], and solitons terminated by half-quantum vortices observed in spinor Bose condensate with quadratic Zeeman energy [38,39]. The $\pi_{2}\left(R_{1}, R_{2}\right)$ describes linear topological solitons and skyrmions terminated by monopoles.

(ii) Another example of the two-manifold system is when the boundary conditions restrict the order parameter on the boundary, with $R_{1}$ being the space in bulk and $R_{2} \subset R_{1}$ is subspace on the boundary restricted by the boundary conditions. This gives the topological classification of the topological objects on the surface of an ordered system [40], such as boojum [41].

(iii) The two-scale system also emerges when there is the hidden symmetry, which may soften the cores of topological defects [42].

In our case of two successive transitions, two energy scales arise in the vicinity of the second transition. There, the coherence length related to the first symmetry breaking
$G \rightarrow H_{1}$ is much smaller than the coherence length related to the second symmetry breaking $H_{1} \rightarrow H_{2}$. This gives rise to two well-defined vacuum manifolds, $R_{1} \cong G / H_{2}$ and $R_{2} \cong$ $H_{1} / H_{2}$, and allows us to apply the relative homotopy groups $\pi_{n}\left(R_{1}, R_{2}\right)$ for classification of the combined objects: stringmonopole objects (analogs of Nambu monopoles); wall-string objects (analogs of KLS wall); nexus [43], etc. That is because the order parameter fields are mapped into different degenerate vacuum manifolds at different spatial regions, thus the homotopy classes of order parameters constitute $\pi_{n}\left(R_{1}, R_{2}\right)$ [44]; see details in Appendix A. In superfluid ${ }^{3} \mathrm{He}$, these topological objects live in the vicinity of transition between the polar phase and the polar distorted $B$ phase $(\mathrm{PdB})$.

This paper is organized as follows. In Sec. II we consider the conventional scheme of the symmetry breaking and the vacuum manifolds of different superfluid phases appeared in the successive transitions. The topological defects in these phases, which emerge due to symmetry breaking and are described in terms of the conventional homotopy groups of vacuum manifolds, are considered in Sec. III. In Sec. IV we discuss combined topological objects in the vicinity of the second transition, where the order parameter could be mapped into two different vacuum manifolds with different coherence lengths. We use the relative homotopy groups and corresponding exact sequence of homomorphisms to describe the classes of combined objects, which are topologically stable in the vicinity of the transition. Based on the exact sequence of the homotopy groups, we find the topological stability of the string monopole (string terminated on monopole) and of the KLS string wall (KLS wall bounded by string). The latter has been observed in recent experiments [9]. In Sec. V we discuss vortex skyrmions emerging in the presence of magnetic field, and the nexus object. In Sec. VI we summarize our results and discuss the role of these objects in formation of the numerous superfluid glass states, which may exist in aerogel [45-47].

\section{CONVENTIONAL SYMMETRY BREAKING SCHEME AND VACUUM MANIFOLDS}

The continuous phase transition is understood as spontaneous symmetry breaking by order parameters about a primary symmetry group $G$. In ${ }^{3} \mathrm{He}$ liquid at low temperature, the order parameters space consists of two three-dimensional vector spaces and the phase space. Thus the order parameter is represented by the complex-valued dyadic tensor $A_{\alpha i}$ [25], which transforms under the action of spin, orbital, and phase rotations of the group $G$. Stabilizer of those actions forms the residual symmetry group $H$ of superfluid phase of ${ }^{3} \mathrm{He}$. In our case, the symmetry group $G$ of normal liquid ${ }^{3} \mathrm{He}$ in the "nematically ordered" aerogel with the uniaxial anisotropy is different from that in the bulk ${ }^{3} \mathrm{He}$ [25]. The uniaxial anisotropy in the orientation of aerogel strands in nafen reduces the symmetry under the $\mathrm{SO}_{L}(3)$ group of rotations in the orbital space to the $O_{L}(2)$ subgroup [9]. If the tiny spin-orbit interaction is neglected, the normal phase vacuum has the following symmetries:

$$
O_{L}(2) \times S O_{S}(3) \times U(1) \times T \times P,
$$


where $S O_{S}(3)$ is the group of spin rotations; $U(1)$ is the global gauge group, which is broken in superfluid states; $T$ is time reversal symmetry; $P$ is parity; $O_{L}(2) \cong S O_{L}(2) \rtimes C_{2 x}^{L}$ where $C_{2 x}^{L}$ is $\pi$ rotation in orbital space.

In what follows, we ignore the time reversal symmetry, since it is not broken in the polar and in $\mathrm{PdB}$ phases, and also ignore the parity $P$ which is reduced to $P e^{i \pi}$ in all $p$-wave superfluid phases, where $e^{i \pi}$ is the $\pi$ rotation in phase space. Also, because we focus on the topological objects related to the spin and $U(1)$ gauge parts of the order parameter, the $\mathbb{Z}_{2}$ symmetry coming from $C_{2 x}^{L}$ could be neglected in the rest of the parts. Then the relevant starting group $G$ of the symmetry breaking scheme in this paper is

$$
G \cong S O_{L}(2) \times S O_{S}(3) \times U(1) .
$$

Starting from this normal phase vacuum, we discuss three types of phase transition: (a) from the normal phase to the polar phase; (b) from the polar phase to the PdB phase; and (c) the possible direct transition from the normal phase to the PdB phase. In this section we consider the topological objects related to these symmetry breaking scenarios, using the conventional homotopy group approach.

\section{A. Transition from normal phase to polar phase}

The order parameter in the $p$-wave spin-triplet superfluids is the dyadic tensor $A_{\alpha i}$, which transforms as a vector under spin rotation (the Greek index) and as a vector under orbital rotations (the Latin index). In the polar phase it has the form:

$$
A_{\alpha i}^{P}=\Delta_{P} \hat{d}_{\alpha} \hat{z}_{i} e^{i \Phi},
$$

where $\Phi$ is the phase, $\hat{d}_{\alpha}(\equiv \hat{\mathbf{d}})$ and $\hat{z}_{i}$ are unit vectors of spin and orbital uniaxial anisotropy, respectively, and $\Delta_{P}$ is the gap amplitude. The residual symmetry group of the polar phase, the symmetry group of the order parameter (3), is

$$
H_{\mathrm{P}} \cong S O_{L}(2) \times S O_{S}(2) \rtimes \mathbb{Z}_{2}^{S-\Phi} \subset G .
$$

Here $\mathbb{Z}_{2}^{S-\Phi} \cong\left\{1, C_{2 x}^{S} e^{i \pi}\right\}$, where $C_{2 x}^{S}$ is the $\pi$ rotation of the vector $\hat{\mathbf{d}}$ about perpendicular axis and $e^{i \pi}$ is the phase rotation by $\pi$, i.e., $\Phi \rightarrow \Phi+\pi$. Then the vacuum manifold of the polar phase is given as

$$
R_{\mathrm{P}} \cong G / H_{\mathrm{P}} \cong\left(S^{2} \times U(1)\right) / \mathbb{Z}_{2} .
$$

The coherence length $\xi=v_{F} / \Delta_{P}$ in the polar phase is the smallest length scale in our problem, which determines the size of singular (hard core) topological defects in the polar phase.

\section{B. From polar phase to $\mathrm{PdB}$ phase}

Let us now consider the second symmetry breaking phase transition: from the polar phase vacuum with fixed $\hat{\mathbf{d}}$ and $\Phi$ to the PdB phase. In the vicinity of this transition the order parameter (3) acquires the symmetry breaking term with amplitude $q \ll 1$ :

$$
A_{\alpha i}^{P d B}=e^{i \Phi} \Delta_{P}\left[\hat{d}_{\alpha} \hat{z}_{i}+q\left(\hat{\mathbf{e}}_{\alpha}^{1} \hat{x}_{i}+\hat{\mathbf{e}}_{\alpha}^{2} \hat{y}_{i}\right)\right] .
$$

Here $\hat{\mathbf{e}}^{1}, \hat{\mathbf{e}}^{2}$, and $\hat{\mathbf{d}}$ form the triad of orthogonal vectors in spin space. The corresponding coherence length of the second transition $\xi / q$ is large in the vicinity of this transition. This provides the hierarchy of the length scales, $\xi$ and $\xi / q \gg \xi$.

The residual symmetry subgroup of the PdB phase in the symmetry breaking from the polar phase is

$$
H_{\mathrm{PdB}} \cong S O_{J}(2) \subset H_{\mathrm{P}},
$$

where $S O_{J}(2)$ represents the common rotations of spin and orbital spaces. The manifold of the vacuum states, which characterizes the second symmetry breaking is

$$
\begin{aligned}
R_{2} & \equiv R_{\mathrm{P} \rightarrow \mathrm{PdB}} \cong H_{\mathrm{P}} / H_{\mathrm{PdB}} \\
& \cong\left(S O_{L}(2) \times S O_{S}(2) \rtimes \mathbb{Z}_{2}^{S-\Phi}\right) / S O_{J}(2) \\
& \cong S O_{L-S}(2) \times \mathbb{Z}_{2}^{S-\Phi} .
\end{aligned}
$$

Here $S O_{L-S}(2)$ is the broken symmetry with respect to relative rotations of spin and orbital spaces.

\section{From normal phase to PdB phase}

Here we consider the situation deep inside the PdB phase, where the parameter $q$ is not necessarily small. In this general case there is only a single length scale which is relevant, and thus this situation becomes similar to that of the direct transition from the normal state to the PdB phase, $G \rightarrow H_{\mathrm{PdB}}$. The order parameter Eq. (6) of the PdB phase could be written as

$$
A_{\alpha i}^{P d B}=e^{i \Phi}\left[\Delta_{\|} \hat{d}_{\alpha} \hat{z}_{i}+\Delta_{\perp}\left(\hat{\mathbf{e}}_{\alpha}^{1} \hat{x}_{i}+\hat{\mathbf{e}}_{\alpha}^{2} \hat{y}_{i}\right)\right],
$$

where $\Delta_{\perp} \leqslant \Delta_{\|}$. The corresponding residual symmetry subgroup is in Eq. (7), and the vacuum manifold of PdB phase in this scenario of symmetry breaking is

$$
R_{1} \equiv R_{\mathrm{normal} \rightarrow \mathrm{PdB}} \cong G / H_{P d B} \cong S O_{L-S}(3) \times U(1) .
$$

\section{TOPOLOGICAL OBJECTS DUE TO SYMMETRY BREAKING TRANSITIONS FROM DIFFERENT VACUA}

In this section we consider the topologically stable defects, which emerge at each of three symmetry breaking transitions discussed in Sec. II.

\section{A. Defects in polar phase due to transition from the normal phase vacuum}

The polar phase vacuum manifold Eq. (5) has the homotopy groups,

$$
\pi_{1}\left(R_{\mathrm{P}}\right) \cong \tilde{\mathbb{Z}}, \pi_{2}\left(R_{\mathrm{P}}\right) \cong \mathbb{Z}, \pi_{0}\left(R_{\mathrm{P}}\right) \cong 0 .
$$

The group $\pi_{1}\left(R_{\mathrm{P}}\right) \cong \tilde{\mathbb{Z}}=\{n / 2 \mid n \in \mathbb{Z}\}$ includes the integers of the group $\mathbb{Z}$ via the inclusion map: $n \in \mathbb{Z} \hookrightarrow n \in \tilde{\mathbb{Z}}$, which describes the conventional quantized vortices with the integer winding number, and also the set of half-odd integers, i.e., $\{n+1 / 2 \mid n \in \mathbb{Z}\}$. The set of half-odd integers describes vortices containing HQV, which has one-half circulation of a conventional quantized vortex. The HQVs with the topological charges $N= \pm 1 / 2$ are the analogs of the cosmological Alice strings.

The group $\pi_{2}\left(R_{\mathrm{P}}\right)=\mathbb{Z}$ describes the hedgehogs (monopoles) in the $\hat{\mathbf{d}}$ field; see Fig. 1. The core size of vortices 
(a)

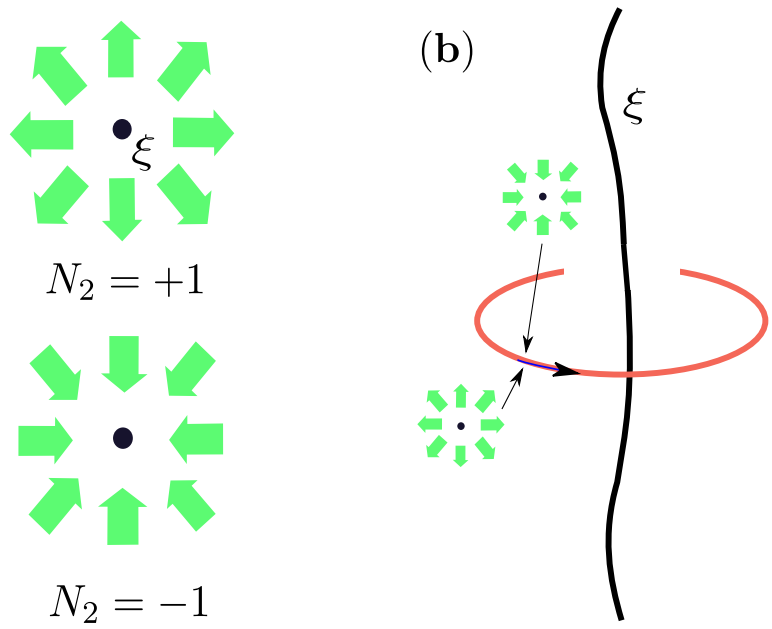

FIG. 1. Topological defects in the polar phase, which exist in addition to the conventional vortices of group $\mathbb{Z}$ with integer circulation quanta. (a) $\hat{\mathbf{d}}$-vector monopole and antimonopole described by the homotopy group $\pi_{2}\left(R_{P}\right)$ with topological charges $N_{2}= \pm 1$ correspondingly. Their core sizes are on the order of the coherence length $\xi$. The green arrows depict the configuration of $\hat{\mathbf{d}}$ vectors. (b) Half-quantum vortex (HQV) described by the $\mathbb{Z}_{2}$ subgroup of $\pi_{1}\left(R_{P}\right)$ with core size $\sim \xi$. This object also got the name "Alice string," because the $\hat{\mathbf{d}}$ monopole transforms to the antimonopole after going around the HQV, in the same manner as it happens for the charge going around the Alice string [49]. The red circle shows the path. Both defects lose topological stability after transition to the PdB phase. The HQV becomes the termination line of the KLS wall bounded by string, and the monopole hedgehog becomes the termination point of spin vortices.

and monopoles is on the order of the coherence length $\xi$. The topological classification of hedgehogs is modified by the phenomenon of influence of the homotopy group $\pi_{1}(R)$ on the group $\pi_{2}(R)$ [48]. The monopole transforms to antimonopole when circling around the Alice string (HQV), and thus in the presence of HQVs the hedgehogs (monopoles) of the group $\mathbb{Z}$ is reduced to the group $\mathbb{Z}_{2}$.

In the polar phase, the HQVs have been identified in NMR experiments [19]. By applying magnetic field tilted with respect to nafen strands, one creates the soliton attached to the HQVs, which produces the measured frequency shift in the NMR spectrum. Hedgehogs (monopoles) are still not identified in superfluid ${ }^{3} \mathrm{He}$.

\section{B. Defects in PdB phase due to transition from normal phase vacuum}

The vacuum manifold $R_{1}$ of the PdB phase in Eq. (10) has homotopy groups,

$$
\pi_{1}\left(R_{1}\right) \cong \mathbb{Z} \times \mathbb{Z}_{2}, \pi_{2}\left(R_{1}\right) \cong 0, \pi_{0}\left(R_{1}\right) \cong 0 .
$$

From all the defects of the polar phase with coherence length size $\xi$ in Sec. III A, only the integer-quantized vortices of group $\mathbb{Z}$ survive in the PdB phase. The other hard core defects (HQVs and hedgehogs) are not supported by topology any more. However, the new topological object appears-the $\mathbb{Z}_{2}$ spin vortex, which becomes topologically stable in the PdB phase. This spin vortex is similar to that which has been observed in the bulk $B$ phase [37].

\section{Defects in PdB phase due to transition from polar phase vacuum}

The vacuum manifold $R_{2}$ of the PdB phase emerging at the transition from the polar phase in Eq. (8) has homotopy groups:

$$
\pi_{1}\left(R_{2}\right) \cong \mathbb{Z}, \pi_{2}\left(R_{2}\right) \cong 0, \pi_{0}\left(R_{2}\right) \cong \mathbb{Z}_{2} .
$$

These homotopy groups are responsible for the topological defects formed in the symmetry breaking transition from the fixed degenerate vacuum of the polar phase (with $\hat{\mathbf{d}}=$ const and $\Phi=$ const) to the PdB phase. Let us consider them separately.

\section{Spin vortices}

The homotopy group $\pi_{1}\left(R_{2}\right) \cong \mathbb{Z}$ describes the spin vortices with $2 \pi n_{1}$ rotation of vectors $\hat{\mathbf{e}}^{1}$ and $\hat{\mathbf{e}}^{2}$ about the fixed $\hat{\mathbf{d}}$ vector of the polar phase. The winding number is

$$
n_{1}=\frac{1}{2 \pi} \oint d x^{i} \hat{\mathbf{e}}^{1} \cdot \nabla_{i} \hat{\mathbf{e}}^{2} .
$$

In the vicinity of the transition, these spin vortices have the soft core of size of the coherence length, which corresponds to the transition from the polar to the PdB phase. This is $\xi / q \gg \xi$. As distinct from the topological defects in the polar phase, which have the "normal" core, the spin vortices in the PdB phase with $R_{2}$ have the "polar" core (quotation marks mean that in multicomponent systems the order parameter is not necessarily equal to zero on the axis of the topological defects). Proliferation of spin vortices in PdB marks the transition to the polar phase.

As follows from Sec. III B, deep in the PdB phase only the $\mathbb{Z}_{2}$ spin vortices survive. The other spin vortices lose the topological stability and thus can live only in the vicinity of the transition from the polar phase vacuum. Far from transition between polar to PdB phase, their topological stability can be restored by applying the magnetic field. In this case spin vortices have the $\hat{\mathbf{d}}$ skyrmions in the core, if the winding number is even (say, doubly quantized spin vortices). The skyrmions in the $\hat{\mathbf{d}}$ field are described by the relative $\pi_{2}$ group and thus are the combined topological objects. All this is discussed in detail in Secs. IV and V.

\section{The fate of monopoles and half-quantum vortices in the PdB phase}

Since in the PdB phase $\pi_{2}\left(R_{1}\right) \cong \pi_{2}\left(R_{2}\right) \cong 0$, the polar phase hedgehog (monopole) is topologically unstable. It becomes the termination point of the spin vortex with two quanta (or the nexus with two singly quantized spin vortices), as discussed in detail in Sec. IV. As a result the $\hat{\mathbf{d}}$ hedgehog becomes the analog of the Nambu monopole, which terminates the electroweak cosmic string [1]; see Fig. 2(b). The analog of the electroweak string in the PdB phase is served either by the doubly quantized spin vortex with $n_{1}=2$, or by the pair of $n_{1}=1$ spin vortices. In the presence of magnetic field, the hedgehog (monopole) separates the string on one 

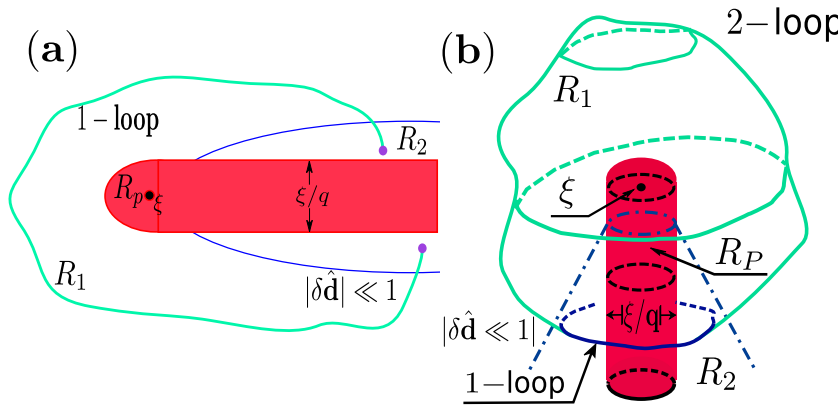

FIG. 2. Illustration of topology describing the combined defects, which emerge in the two-step transition: from the normal state to the polar phase and then from the polar phase to the polar distorted $B$ phase. (a) KLS string wall. In general the KLS wall is nontopological, but it acquires the nontrivial topology in the vicinity of the second phase transition. In this limit case there are two well-separated length scales: the coherence length $\xi$ of the first transition, which determines the size of the hard core of string (the black dot), and the much larger coherence length $\xi / q \gg \xi$ of the second transition, which determines the soft core size of the wall (the pink region). The hierarchy of scales gives rise to two types of the degenerate vacua in the $\mathrm{PdB}$ phase, $R_{1}$ and $R_{2}$. The $R_{1}$ vacua include all the degenerate vacua of the PdB phase, while the $R_{2}$ vacua are those, which are obtained from the fixed order parameter of the polar phase, i.e., at fixed $\hat{\mathbf{d}}$ and $\Phi$ in Eq. (3). This is the region, where the asymptotic condition $|\delta \hat{\mathbf{d}}| \ll 1$ is achieved. The blue line shows the characteristic border between the regions of two classes of vacuum spaces. The topology of the string wall is determined by the relative homotopy group $\pi_{1}\left(R_{1}, R_{2}\right)$, in which the green loop is mapped to the space $R_{1}$, with the ends of the loop mapped to $R_{2}$. (b) The string monopole is described by the relative homotopy group $\pi_{2}\left(R_{1}, R_{2}\right)$. In this case the black dot shows the core of the hedgehog in the $\hat{\mathbf{d}}$ field and the pink region is the core of $4 \pi$ spin vortex, which is terminated by the hedgehog. The green two-loop edge is mapped to the space $R_{1}$, with its one-loop edge mapped to $R_{2}$.

side of the monopole and the skyrmion on the other side of the monopole.

\section{The fate of half-quantum vortices in the PdB phase and the KLS wall}

A similar situation takes place with the HQVs, which are not topologically stable in the PdB phase. They become the termination lines of the KLS cosmic walls, as discussed in Sec. IV; see Fig. 2(a). In ${ }^{3} \mathrm{He}$ experiments, after transition from the polar phase to the $\mathrm{PdB}$ phase in the presence of HQVs, the KLS walls appear between the neighboring vortices, and in spite of the tension of domain walls, the HQVs remain pinned by the nafen strands [9].

In general the KLS wall is not topologically stable, and can be stabilized only due to symmetry reasons [50]. However, in the vicinity of the transition to $\mathrm{PdB}$ phase from the polar phase vacuum, the KLS wall becomes topological. The topological domain wall of the thickness $\xi / q$ is described by the nonzero element of the homotopy group $\pi_{0}\left(R_{\mathrm{P} \rightarrow \mathrm{PdB}}\right) \cong \mathbb{Z}_{2}$. An example of such a wall is the domain wall between the domains with $A_{\alpha i}=\Delta_{P} \operatorname{Diag}(1, q, q)$ and $A_{\alpha i}=\Delta_{P} \operatorname{Diag}(1, q,-q)$.

\section{COMBINED OBJECTS}

\section{A. Combined objects and classification by relative homotopy groups}

As mentioned before, in the vicinity of the second transition, the system has two different length scales, $\xi$ and $\xi / q \gg \xi$. This leads to the new classes of objects, which combine the topology of both vacuum spaces $R_{1}$ and $R_{2}$. Such combined objects are described by the relative homotopy groups $[34,40,42,44]$,

$$
\pi_{n}\left(R_{1}, R_{2}\right) \text {. }
$$

In particular, some elements of group $\pi_{1}\left(R_{1}, R_{2}\right)$ describe the string wall in Fig. 2(a) (the wall bounded by strings, such as the KLS wall [2]), while the nontrivial elements of the group $\pi_{2}\left(R_{1}, R_{2}\right)$ describe string monopoles in Fig. 2(b) (such as the string terminated by the Nambu monopole [1]). These combined objects are topologically stable only in the vicinity of the second transition, and they lose topological stability when two length scales become comparable.

This combined topology can be illustrated by the following example of the string wall. At small distances $\xi \ll r \ll \xi / q$ from the core of HQV, the HQV is described by the homotopy group $\pi_{1}\left(R_{P}\right)$. However, at larger distances $r \gg \xi / q$, the HQV becomes the termination line of the wall, which is described by the $\pi_{0}\left(R_{2}\right)$ topology; see Fig. 2(a). This figure demonstrates distributions of the degeneracy spaces $R_{1}$ and $R_{2}$, which are involved in the topology of the combined object.

The similar physics takes place for string monopoles. At small distances $\xi \ll r \ll \xi / q$ from the core of the hedgehog, it is described by the homotopy group $\pi_{2}\left(R_{P}\right)$, while at larger distances $r \gg \xi_{q}$, the monopole becomes the termination point of spin vortices described by the $\pi_{1}\left(R_{2}\right)$ topology; see Fig. 2(b). Such combinations of $\pi_{n+1}$ and $\pi_{n}$ groups needed for the description of the object with two different length scales and two different dimensions are the relative homotopy groups.

The relative homotopy groups $\pi_{n}\left(R_{1}, R_{2}\right)$ can be found from the following consideration. Since $R_{1}=G / H_{P d B}$ and $R_{2}=H_{P} / H_{P d B}$, one has $R_{1} / R_{2}=\left(G / H_{P d B}\right)\left(H_{P} / H_{P d B}\right)=$ $G / H_{P}=R_{P}$, and thus all the elements of the relative homotopy groups $\pi_{n}\left(R_{1}, R_{2}\right)$ are determined by the elements of conventional homotopy groups $\pi_{n}\left(R_{P}\right)$ of the polar phase (we thank the referee for this comment):

$$
\pi_{n}\left(R_{1}, R_{2}\right) \cong \pi_{n}\left(R_{P}\right) .
$$

This relation demonstrates that in the vicinity of the phase transition from the first (polar) phase to the second (PdB) phase, all the topological objects of the first phase described by the group $\pi_{n}\left(R_{P}\right)$ retain their topological charges in the second phase. Some of these defects remain free, while the others become the parts of the composite defects - the string monopole and for the KLS string wall in Secs. IV B and IV C, respectively.

Equation (16) does not resolve between the free and the composite objects of the second phase. The full classification of the topological objects in the second phase depends not only on $R_{1} / R_{2}$, but also on the details of the mappings in the exact sequence of homomorphisms, which is calculated in Appendixes A and B. The mapping diagram of the exact 


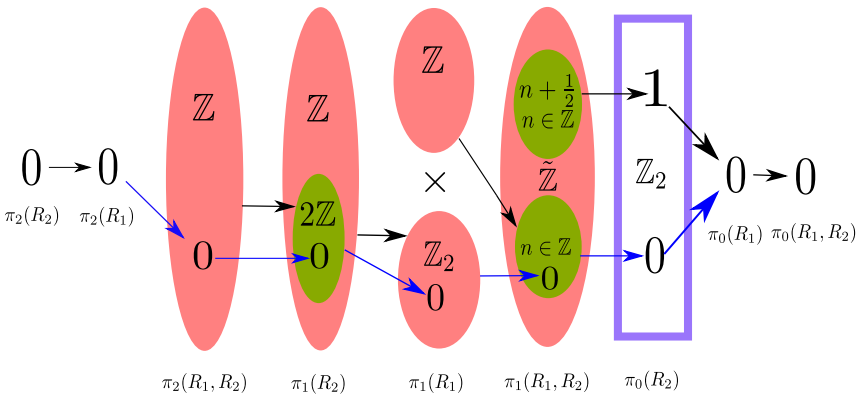

FIG. 3. Illustration of the exact sequence of homomorphisms to the calculation of the relative homotopy groups $\pi_{n}\left(R_{1}, R_{2}\right)$. This demonstrates that the elements of the $\pi_{n}\left(R_{1}, R_{2}\right)$ group have two sources: from the kernel of the mapping $\pi_{n-1}\left(R_{2}\right) \rightarrow \pi_{n-1}\left(R_{1}\right)$ and from the factor group of $\pi_{n}\left(R_{1}\right)$ over the image of the mapping $\pi_{n}\left(R_{2}\right) \rightarrow \pi_{n}\left(R_{1}\right)$. The black arrows represent the image of homomorphisms, while the blue arrows represent the kernal of every homomorphsim. This mapping diagram prescribes the relation between the elements of the composite topological defects. In particular, it shows that the relative homotopy group $\pi_{2}\left(R_{1}, R_{2}\right)$ is determined by the kernel of the mapping $\pi_{1}\left(R_{2}\right) \rightarrow \pi_{1}\left(R_{1}\right)=2 \mathbb{Z} \cong \mathbb{Z}$. It demonstrates that the nontrivial monopoles are termination points of spin vortices with the total winding number being even. As we will see in the next section, this mapping relation is identical to that describing vortex skyrmions. On the other hand, the relative homotopy group $\pi_{1}\left(R_{1}, R_{2}\right) \cong \tilde{\mathbb{Z}}$ is determined by both sources: $\mathbb{Z}_{2}$ which is the kernel of the homomorphism $\pi_{0}\left(R_{2}\right) \rightarrow \pi_{0}\left(R_{1}\right)$ and by the quotient group of $\pi_{1}\left(R_{1}\right)$ over the image of homomorphism between $\pi_{1}\left(R_{2}\right)$ and $\pi_{1}\left(R_{1}\right)$. As a result, there are two different kinds of phase vortices that terminate and do not terminate the KLS wall. Those two classes of vortices consist of the two cosets of quotient $\tilde{\mathbb{Z}} / \mathbb{Z} \cong$ $\mathbb{Z}_{2}$. Correspondingly, these are the vortices with half-odd integer circulation numbers and the vortices with integer circulation quanta.

sequence in Fig. 3 depicts the relation between different topological objects in $R_{1}$ and $R_{2}$.

\section{B. Wall bounded by string-KLS string wall}

The relative homotopy group is

$$
\pi_{1}\left(R_{1}, R_{2}\right) \cong \pi_{1}\left(R_{P}\right) \cong \tilde{\mathbb{Z}} .
$$

This shows that the topological charges of vortices in the second $(\mathrm{PdB})$ phase are the same as in the first (polar) phase. In both phases they form the group of integer and halfodd integers $N$. However, the physical realizations of these vortices are different in the two phases. Vortices with integer $N$ remain free, while vortices with half-odd integers, $N=k+$ $1 / 2$, terminate the wall bounded by string - the KLS string wall. Figure 4 illustrates the configuration of the composite object. This kind of topologically protected KLS string wall induces the cosmological catastrophe in the axion solution of the strong $\mathrm{CP}$ problem [6-8].

In general, in the vicinity of the second phase transition the topological objects of the first phase remain topological in the second phase. Some of these defects remain free, while the others become the part of the composite objects with the same topological invariants. The separation between these two groups of objects is determined by the mappings in the exact sequence of homomorphisms.

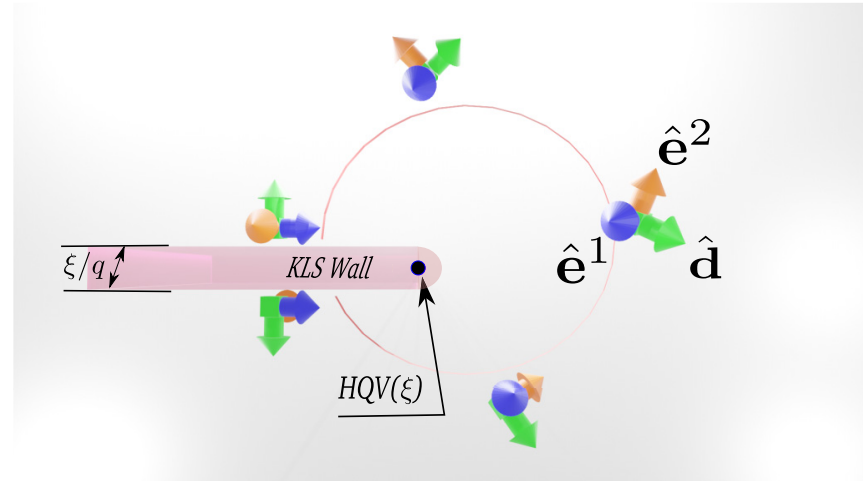

FIG. 4. Illustration of the KLS string wall. The wall is topologically protected if the order parameter takes values from disconnected parts of $R_{2}$. In this case it is the combined object-the HQV, which terminates the KLS wall. The pink region is the topological KLS wall with thickness $\xi / q$, while the black small dot is the HQV string, in which the diameter is $\xi$. The spin tripods show the configurations of order parameter around the HQV string. The flipping of the tripods on two sides of the KLS wall demonstrates the domain wall feature.

\section{Strings terminated by monopole-string monopole}

The relative homotopy group,

$$
\pi_{2}\left(R_{1}, R_{2}\right) \cong \pi_{2}\left(R_{P}\right) \cong \mathbb{Z},
$$

describes monopoles (hedgehogs) of the $\hat{\mathbf{d}}$ field. They survive in the vicinity of the second transition as the topological objects which terminate the spin vortices. The corresponding composite object-the string monopole- has two topological charges, $n_{2}$ and $n_{1}$, which are related as

$$
n_{2}=\frac{1}{8 \pi} e^{i j k} \int_{\mathrm{S}^{2}} d S_{k} \hat{\mathbf{d}} \cdot\left(\nabla_{i} \hat{\mathbf{d}} \times \nabla_{j} \hat{\mathbf{d}}\right)=\frac{1}{2} n_{1} .
$$

Here $S^{2}$ is the surface encircling monopole and the group $\mathbb{Z}$ is the group of integers $n_{2}$-the topological charges of the hedgehog. The $n_{1}$ is the winding number of spin vortices in Eq. (14). The equation $n_{1}=2 n_{2}$ in (19) shows that the monopole can be the termination point of spin vortices with the even total charge $n_{2}$. This situation is similar to the monopole in the chiral A phase [51-53], which either terminates a single vortex with $n_{1}=2$, or forms the nexus with two singly quantized vortices with $n_{1}=1+1=$ 2 , or with four HQVs with $n_{1}=1 / 2+1 / 2+1 / 2+1 / 2=$ 2 . Those vortices, which connect with monopoles $\left(n_{2}>0\right)$ or antimonopoles $\left(n_{2}<0\right)$ allow the existences of complex monopole-antimonopole networks [4,54-56].

Figure 5 illustrates the configuration of the string monopole, which consists of the hedgehog with $n_{2}=1$ and two strings-spin vortices each with $n_{1}=1$. The spin vortices have a soft core with size $\xi / q$.

\section{SKYRMIONS AND NEXUS IN THE PRESENCE OF MAGNETIC FIELD}

In the presence of magnetic field $\mathbf{H}$, a new length scale appears in the PdB phase - the magnetic length $\xi_{H} \propto|\mathbf{H}|^{-1}$. The magnetic length $\xi_{H}$ is the longest length scale if we neglect the spin-orbit coupling. In this case, one obtains the 

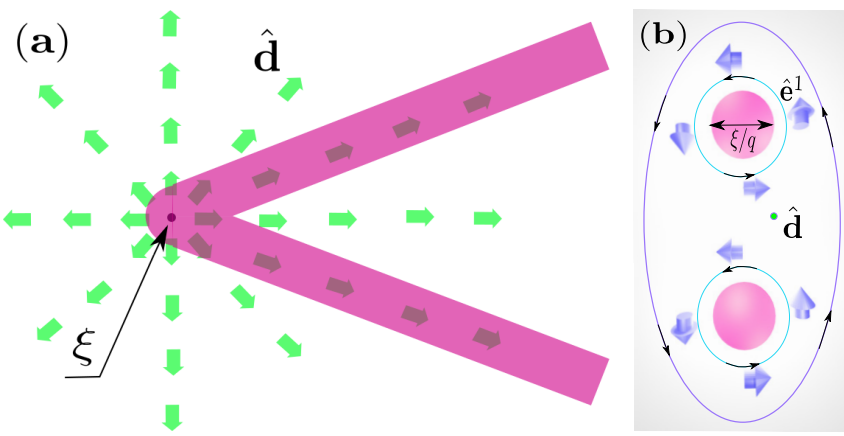

FIG. 5. Illustration of string monopole in the PdB phase. The monopole in the $\hat{\mathbf{d}}$-field has topological charge $n_{2}=1$ and hard core of the coherence length size $\xi$ (black dot). This string monopole terminates two spin vortices with the soft core size $\xi / q$ and with the total topological charge $n_{1}=1+1=2=2 n_{2}$ according to Eq. (19). (a) Texture of $\hat{\mathbf{d}}$ vector string monopole with $n_{2}=2$ terminating two spin vortices. The green arrow corresponds to $\hat{\mathbf{d}}$ vector. The red region is the soft core of spin vortex of size $\xi / q$. (b) The cross sections of two spin vortices with $n_{1}=1$ each. In these cross sections, $\hat{\mathbf{e}}_{1}$ and $\hat{\mathbf{e}}_{2}$ vectors rotate around $\hat{\mathbf{d}}$ vector by $2 \pi$ for every spin vortex. The core size of every spin vortex is $\xi / q$. The blue line is the field line of total $\hat{\mathbf{e}}$ vectors rotation, while the green lines are $\hat{\mathbf{e}}$ vectors field lines of every spin vortex.

two-scale system of type (i) in Introduction. In the region with length scale larger than $\xi_{H}$, the magnetic anisotropy locks the directions of $\hat{\mathbf{d}}$ vector in the plane perpendicular to $\mathbf{H}$ to minimize the magnetic energy, which is proportional to $|\mathbf{H} \cdot \hat{\mathbf{d}}|^{2}$. The degenerate space of the order parameter is reduced from $R_{1} \cong S O_{L-S}(3) \times U(1)$ in Eq. (10) to $R_{1}^{H}=$ $S^{1} \times S^{1} \times U(1)$ in the regions which are larger than $\xi_{H}$. The first $S^{1}$ is the manifold of in plane $\hat{\mathbf{d}}$ vector, while the second $S^{1}$ is the manifold of rotations of $\hat{\mathbf{e}}^{1}$ and $\hat{\mathbf{e}}^{2}$ about the $\hat{\mathbf{d}}$-axis. Then the second relative homotopy group of combined objects with length scale $\xi_{H}$ is $\pi_{2}\left(R_{1}, R_{1}^{H}\right) \cong \mathbb{Z} \times \mathbb{Z}$. However, for $q \ll 1$, the gradient energy of the $\hat{\mathbf{d}}$-textures is much larger than that of the textures in $\hat{\mathbf{e}}^{1}$ and $\hat{\mathbf{e}}^{2}$ fields [25]. That is why we consider only the $S^{1}$ manifold of $\hat{\mathbf{e}}^{1}$ and $\hat{\mathbf{e}}^{2}$, and neglect the $S^{1}$ manifold of $\hat{\mathbf{d}}$. Then the relative second homotopy group which we need in this case is

$$
\pi_{2}\left(R_{1}, S^{1} \times U(1)\right) \cong \mathbb{Z} .
$$

These results for the relative homotopy group have been confirmed by calculations using the exact sequence, see details in Appendixes A and B. The mapping diagram of exact sequence is shown in Fig. 6.

The relative homotopy group $\pi_{2}\left(R_{1}, S^{1} \times U(1)\right)$ describes the composite object in the PdB phase in the presence of magnetic field. This object is the spin vortex with an even winding number, which has the soft core of size $\xi_{H}$ represented as skyrmion (see Fig. 7). The topological charge of skyrmion is

$$
n_{2}=\frac{1}{8 \pi} e^{i j k} \int_{\mathrm{D}_{2}} d S_{k} \hat{\mathbf{d}} \cdot\left(\nabla_{i} \hat{\mathbf{d}} \times \nabla_{j} \hat{\mathbf{d}}\right)=\frac{1}{2} n_{1},
$$

where $D_{2}$ is the cross section of skyrmion and $n_{1}$ is the winding number of spin vortices in Eq. (14). Equation (21) is the analog of the Mermin-Ho relation in ${ }^{3} \mathrm{He}-\mathrm{A}$ [57]. Equation (21) is identical to Eq. (19) because of $\pi_{2}\left(R_{1}, S^{1} \times U(1)\right) \cong$

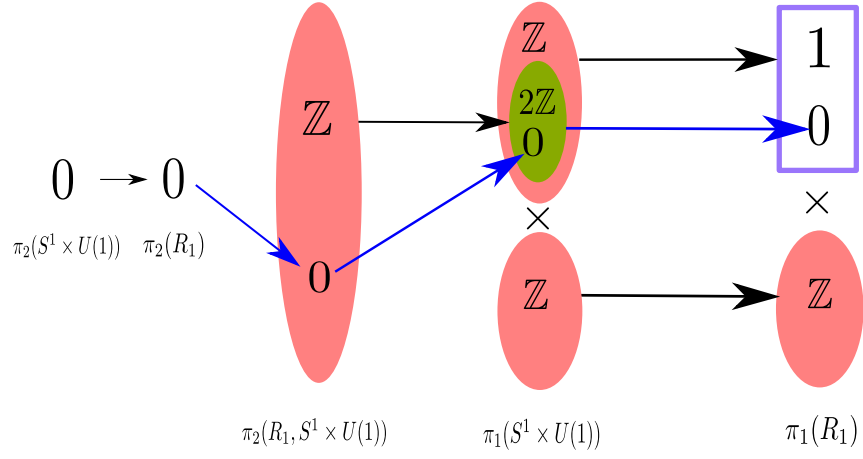

FIG. 6. Mapping diagram of exact sequence between $R_{1}$ and $S^{1} \times U(1)$. The black arrows represent the image of homomorphisms, while the blue arrows represent the kernal of every homomorphsim. This diagram shows that the skyrmions soften the core of $\mathbb{Z}$ spin vortices with size $\xi / q$ to size $\xi_{H}$ in the presence of magnetic field. In regions larger than $\xi_{\mathbf{H}}$, vortex skyrmions can be connected with spin vortices via string monopole, if their total topological charge is even according to Eq. (21). This is because $\pi_{2}\left(R_{1}, R_{2}\right) \cong$ $\pi_{2}\left(R_{1}, S^{1} \times U(1)\right)$. There are also phase vortices described by $\mathbb{Z}$, but here we ignore them because they do not influence the connection between the spin vortices and skyrmions.

$\pi_{2}\left(R_{1}, R_{2}\right)$. Due to this relation the vortex skyrmion can be connected to $\mathbb{Z}$ spin vortices with core size $\xi / q$ via the string monopole. Such composite objects, where the monopole connects several linear objects is called nexus. It demonstrates the interplay between $\pi_{1}$ and $\pi_{2}$ topologies.

Originally vortex skyrmions formed by orbital and phase degenerate parameters have been suggested in ${ }^{3} \mathrm{He}-\mathrm{A}$ by $\mathrm{An}$ derson and Toulouse [58] and by Chechetkin [59]. The lattice of vortex skyrmions in rotating ${ }^{3} \mathrm{He}-\mathrm{A}$ has been discussed in Ref. [60]. These objects have been identified in different experiments made under rotation $[61,62]$. The dynamics of (a) $\hat{\mathrm{d}}$ skyrmion

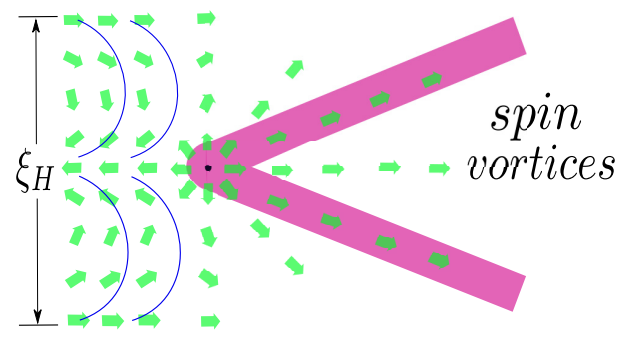

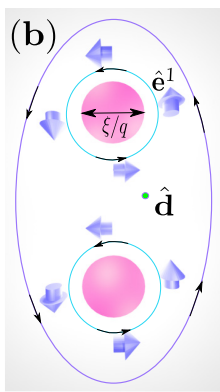

FIG. 7. Illustration of the nexus object in the presence of magnetic field. The nexus connects spin vortices with core size $\xi / q$ and the vortex skyrmion with core size $\xi_{H}$, where $\xi_{H} \gg \xi / q$. (a) The texture configuration of the nexus object with $n_{2}=1$ and $n_{1}=2$. The green arrows are the $\hat{\mathbf{d}}$ vectors and the pink regions are core regions of defects. The black dot is the core of the string monopole with size $\xi$, while the pink regions are the cores of spin vortices with size $\xi / q$. The green arrows represent the distribution of $\hat{\mathbf{d}}$ vectors. The vortex skyrmion with core size $\xi_{H}$ transforms to two spin vortices via the nexus. (b) The cross section of two spin vortices. Every spin vortex has the $2 \pi$ rotation of $\hat{\mathbf{e}}^{1}$ and $\hat{\mathbf{e}}^{2}$ vectors around the fixed $\hat{\mathbf{d}}$ vector. The blue arrows represent the field of the $\hat{\mathbf{e}}^{1}$ vector. 
the vortex skyrmions provides an effective electromagnetic field, which induces the observed effect of chiral anomaly experienced by fermionic excitations (Weyl fermions) living in the soft core of a vortex skyrmion [63].

\section{CONCLUSION AND DISCUSSION}

Here we discussed the topology, which emerges in two-step phase transition in the vicinity of the second transition. An example is provided by the second-order phase transition from the normal ${ }^{3} \mathrm{He}$ to the polar phase followed by the secondorder phase transition from the polar phase to the PdB phase experimentally observed in superfluid ${ }^{3} \mathrm{He}$ in nafen [9]. Here the composite object-the analog of the KLS wall bounded by cosmic string - has been observed. We demonstrated that in the vicinity of the second transition, such composite object is described by the relative homotopy groups. The reason for that is the existence of the two well-separated length scales. The smaller length scale determines the core size of the halfquantum vortex (the analog of Alice cosmic string). It is the coherence length $\xi$ related to the symmetry breaking phase transition from the normal liquid to the polar phase. The larger length scale $\xi / q \gg \xi$ determines the soft core size of the KLS wall terminated by this string. It is the coherence length related to the second symmetry breaking phase transitionthe transition form the polar phase to the PdB phase.

The two-scale composite defects are described by relative homotopy groups $\pi_{n}\left(R_{1}, R_{2}\right)$. Here $R_{1}$ is the vacuum manifold of the PdB phase, while $R_{2}$ is also the vacuum manifold of the PdB phase, but at a fixed value of the order parameter of the polar phase before the transition to the PdB phase. The observed KLS wall terminated by the half-quantum vortex is determined by the nontrivial element of $\pi_{1}\left(R_{1}, R_{2}\right)$. The other composite object, which is still waiting for its observation, is the monopole (hedgehog), which terminates the string (the spin vortex). Its topology is determined by the nontrivial element of $\pi_{2}\left(R_{1}, R_{2}\right)$. The core of the monopole is of coherence length size $\xi$, while the spin vortices have the soft core of size $\xi / q \gg \xi$. The relative homotopy groups $\pi_{n}\left(R_{1}, R_{2}\right)$ are calculated using the exact sequence of the group homomorphisms.

The topology of these combined objects demonstrates new application of the relative homotopy groups. Earlier the relative homotopy groups have been applied for classification of topological defects on the surface of the ordered system [40], and for classification of topological solitons terminated by point or linear defects [34].

We also considered the more complicated object-the nexus, which combines the monopole, the string terminated by monopole, and skyrmion (topological soliton) terminated by the same monopole. Such object in the PdB phase arises in the presence of magnetic field, which provides another length scale. The situation becomes even richer, when the spin-orbit interaction is included, which provides the fourth length scale and extends the multiscale topology. The objects combining vortices and skyrmions were recently considered for superconductor-ferromagnet heterostructures, in which the existence of Majorana bound states were suggested [64,65].

Typically the state of the system with topological defects represents the excited state of the system. However, the topological defects can form the ground state. Earlier it was suggested that the suppression of the $B$ phase on the boundary of superfluid ${ }^{3} \mathrm{He}$ may lead to formation of the stripe phase in superfluid ${ }^{3} \mathrm{He}-\mathrm{B}$ under nanoscale confinement in a slab geometry [66]. On a microscopic level, this inhomogeneous phase is thought as the periodic array of the KLS domain walls between the degenerate states of the $B$ phase; see Refs. [32,50]. The possible observation of such spatially modulated phase has been reported [67,68]. A similar situation may take place in another kind of confined geometry, in nafen. The strands of nafen could play the same role as the boundaries in the slab confinement. The suppression of the order parameter near the strands may result in the spontaneous proliferation of the composite defects leading to the stripe phases or stripe glasses.

\section{ACKNOWLEDGMENTS}

We thank Jaakko Nissinen and Ilya M. Eremin for discussions. This work has been supported by the European Research Council (ERC) under the European Union's Horizon 2020 research and innovation programme (Grant Agreement No. 694248).

\section{APPENDIX A: EXACT SEQUENCES: WITH AND WITHOUT MAGNETIC FIELD}

\section{No magnetic field}

The exact sequence of (relative) homotopy groups means that the image of any homomorphism $x_{*}^{n}: A \rightarrow B$ (the sets of the elements of group $B$ into which the elements of $A$ are mapped) is the kernel of the next homomorphism $x_{*}^{n+1}$ : $B \rightarrow C$ (the sets of the elements of $B$ which are mapped to the zero element of $C$ ) i.e., $\operatorname{im} x_{*}^{n} \cong \operatorname{ker} x_{*}^{n+1}$, with $n \in$ $\mathbb{Z}$ [69]. The relative homotopy classes of $\pi_{k+1}\left(R_{1}, R_{2}\right)$ are mapped to the homotopy classes of $\pi_{k}\left(R_{2}\right)$ by mapping the $k$-dimension subset of the $k+1$ sphere, which surrounds the defects, into $R_{2}$. This mapping between two homotopy classes with different dimensions is called boundary homomorphism $\partial_{*}$ [69]. Boundary homomorphism shows how topological objects with different dimensions connect to each other. In the $\mathrm{PdB}$ phase the exact sequence of homomorphisms is

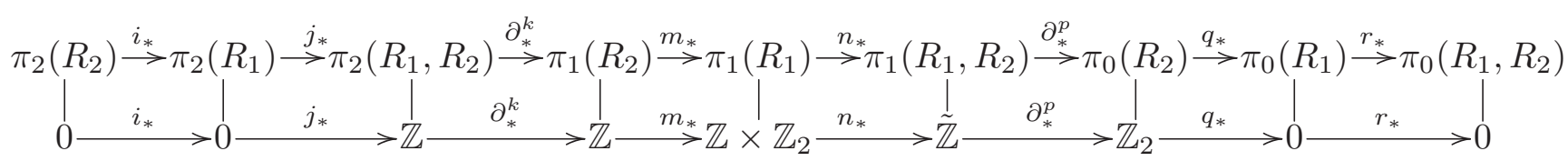


where the $\partial_{*}^{k}$ and $\partial_{*}^{p}$ are boundary homomorphisms. This gives the following relative homotopy groups: $\pi_{2}\left(R_{1}, R_{2}\right) \cong \mathbb{Z}$,

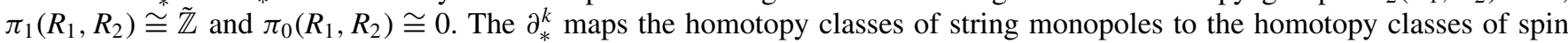
vortices. The $\partial_{*}^{p}$ maps the homotopy classes of the KLS string wall to homotopy classes of the domain wall. The kernels and images of every relative homotopy group are analyzed in Appendix B.

\section{In the presence of magnetic field}

In the presence of magnetic field the corresponding exact sequence is

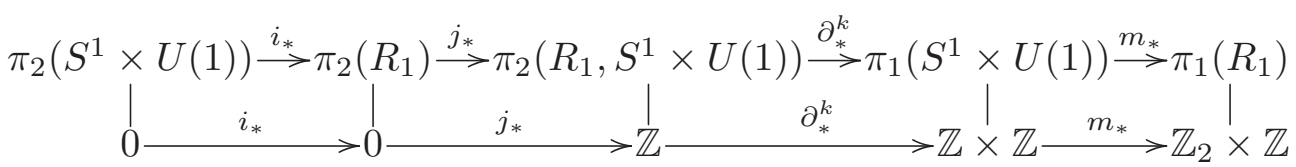

i.e., $\pi_{2}\left(R_{1}, S^{1} \times U(1)\right)=2 \mathbb{Z} \cong \mathbb{Z}$. We found $\operatorname{ker} \partial_{*}^{k} \cong 0$ and $\operatorname{im} \partial_{*}^{k}=2 \mathbb{Z} \cong \mathbb{Z}$. That means that only those objects are topological protected, which have an even total winding number of spin rotation. These objects are the $\hat{\mathbf{d}}$-vector skyrmions. Since $\pi_{2}\left(R_{1}, S^{1} \times U(1)\right) \cong \pi_{2}\left(R_{2}, R_{1}\right)$, these $\hat{\mathbf{d}}$ skyrmions can terminate on the $\hat{\mathbf{d}}$ monopole, which in turn is the end point of spin vortices with the total even number of spin rotation. As a result one obtains the composite effect- the nexus in Fig. 7.

\section{APPENDIX B: RELATIVE HOMOTOPY GROUPS}

$$
\text { 1. } \pi_{2}\left(R_{1}, R_{2}\right)
$$

The objects described by the $\pi_{2}\left(R_{1}, R_{2}\right)$ are monopoles of the $\hat{\mathbf{d}}$ vector, because the manifold of the degenerate states of the $\hat{\mathbf{d}}$ vector is $S^{2} \cong S O_{S}(3) / S O_{S}(2)$, and we have the mapping from $S^{2}$ in real space to the $S^{2}$ manifold of $\hat{\mathbf{d}}$ vectors.

The boundary homomorphism $\partial_{*}^{k}$ maps $S^{1} \subset S^{2}$ to $R_{2}$. Then the im $\partial_{*}^{k}$ describes all classes of string defects terminated by the monopoles. We found $\operatorname{im} \partial_{*}^{k}=2 \mathbb{Z} \cong \mathbb{Z}$ which is the set of even numbers. This means that only the spin vortices with the total even winding number can form the string monopole. This situation is similar to the monopole connected with four half-quantum vortices in the A phase, where the total winding number is 2 [51]. The topologically trivial monopole cannot connect with the string defects be- cause of $\operatorname{ker} \partial_{*}^{k} \cong 0$. Actually this trivial class is identical to $\pi_{2}\left(R_{1}\right)$ because $\operatorname{ker} j_{*} \cong \operatorname{im} i_{*} \cong 0$.

$$
\text { 2. } \pi_{1}\left(R_{1}, R_{2}\right)
$$

The relative homotopy group is $\pi_{1}\left(R_{1}, R_{2}\right) \cong \tilde{\mathbb{Z}}$. From $\operatorname{ker} q_{*} \cong \operatorname{im} \partial_{*}^{p} \cong \pi_{0}\left(R_{2}\right) \cong \mathbb{Z}_{2}$, we know there are domain walls bounded by string defects. The set of half-odd integers of the group $\tilde{\mathbb{Z}}$, which come from im $\partial_{*}^{p}$ describes the domain wall terminated by string defects-the KLS wall terminated by HQV or by any vortex with half-odd integer winding number $N=k+1 / 2$. The vortices, which come from $\operatorname{ker} \partial_{*}^{p} \cong \mathbb{Z}$ are vortices with the integer winding number. These vortices are free.

$$
\text { 3. } \pi_{1}\left(R_{1}, S^{1} \times U(1)\right)
$$

From the exact sequence in Eq. (A2) it follows that $\pi_{2}\left(R_{1}, S^{1} \times U(1)\right)=2 \mathbb{Z} \cong \mathbb{Z}$. This group describes the linear skyrmions in the $\hat{\mathbf{d}}$ vector, which are the linear analogs of the original pointlike skyrmion [70,71]. The spin texture inside the cross section $D_{2}$ of the skyrmion corresponds to continuous mapping to $\mathrm{SO}_{S}(3)$, which is implemented by choosing first a direction of $\hat{\mathbf{d}}$ and then making the $S O_{S}(2)$ rotation of $\hat{\mathbf{e}}^{1}$ and $\hat{\mathbf{e}}^{2}$ around this direction. This skyrmion also represents the spin vortex with an even winding number, because of im $\partial_{*}^{k} \cong 2 \mathbb{Z}$ and $\operatorname{ker} \partial_{*}^{k} \cong 0$.
[1] Y. Nambu, String-like configurations in the Weinberg-Salam theory, Nucl. Phys. B 130, 505 (1977).

[2] T. W. B. Kibble, G. Lazarides, and Q. Shafi, Walls bounded by strings, Phys. Rev. D 26, 435 (1982); Strings in SO(10), Phys. Lett. B 113, 237 (1982).

[3] T. W. B. Kibble, Classification of topological defects and their relevance to cosmology and elsewhere, in Topological Defects and the Non-Equilibrium Dynamics of Symmetry Breaking Phase Transitions, NATO Science Series (Series C: Mathematical and Physical Sciences), Vol. 549, edited by Y. M. Bunkov and H. Godfrin (Springer, Dordrecht, 2000), pp. 7-31.

[4] T. W. B. Kibble and T. Vachaspati, Monopoles on strings, J. Phys. G 42, 094002 (2015).

[5] A. Vilenkin and A. E. Everett, Cosmic Strings and Domain Walls in Models with Goldstone and Pseudo-Goldstone Bosons, Phys. Rev. Lett. 48, 1867 (1982).
[6] P. Sikivie, Axions, Domain Walls, and the Early Universe, Phys. Rev. Lett. 48, 1156 (1982).

[7] A. Caputo and M. Reig, Cosmic implications of a low-scale solution to the axion domain wall problem, Phys. Rev. D 100, 063530 (2019).

[8] C. Chatterjee, T. Higaki, and M. Nitta, Note on a solution to domain wall problem with the Lazarides-Shafi mechanism in axion dark matter models, Phys. Rev. D 101, 075026 (2020).

[9] J. T. Mäkinen, V. V. Dmitriev, J. Nissinen, J. Rysti, G. E. Volovik, A. N. Yudin, K. Zhang, and V. B. Eltsov, Half-quantum vortices and walls bounded by strings in the polar-distorted phases of topological superfluid ${ }^{3} \mathrm{He}$, Nat. Comm. 10, 237 (2019).

[10] K. Aoyama and R. Ikeda, Pairing states of superfluid ${ }^{3} \mathrm{He}$ in uniaxially anisotropic aerogel, Phys. Rev. B 73, 060504 (2006). 
[11] S. Yang and R. Ikeda, Possibility of unconventional pairing states in superfluid ${ }^{3} \mathrm{He}$ in uniaxially anisotropic aerogels, J. Phys. Soc. Jpn 83, 084602 (2014).

[12] R. Sh. Askhadullin, V. V. Dmitriev, D. A. Krasnikhin, P. N. Martynov, A. A. Osipov, A. A. Senin, and A. N. Yudin, Phase diagram of superfluid ${ }^{3} \mathrm{He}$ in "nematically ordered" aerogel, JETP Lett. 95, 326 (2012).

[13] P. W. Anderson, Theory of dirty superconductors, J. Phys. Chem. Solids 11, 26 (1959).

[14] I. A. Fomin, Analog of Anderson theorem for the polar phase of liquid ${ }^{3} \mathrm{He}$ in nematic aerogel, JETP 127, 933 (2018).

[15] I. A. Fomin, Temperature dependence of the order parameter of the polar phase of liquid ${ }^{3} \mathrm{He}$ in nematic aerogel, arXiv:2003.9652.

[16] M. Tange and R. Ikeda, Half-quantum vortex pair in polardistorted B phase of superfluid ${ }^{3} \mathrm{He}$ in aerogels, Phys. Rev. B. 101, 094512 (2020).

[17] A. Ramires, D. F. Agterberg, and M. Sigrist, Tailoring $T_{c}$ by symmetry principles: The concept of superconducting fitness, Phys. Rev. B 98, 024501 (2018).

[18] V. B. Eltsov, T. Kamppinen, J. Rysti, and G. E. Volovik, Topological nodal line in superfluid ${ }^{3} \mathrm{He}$ and the Anderson theorem, arXiv:1908.1645.

[19] S. Autti, V. V. Dmitriev, J. T. Mäkinen, A. A. Soldatov, G. E. Volovik, A. N. Yudin, V. V. Zavjalov, and V. B. Eltsov, Observation of Half-Quantum Vortices in Superfluid ${ }^{3} \mathrm{He}$, Phys. Rev. Lett. 117, 255301 (2016).

[20] G. E. Volovik and V. P. Mineev, Line and point singularities in superfluid ${ }^{3} \mathrm{He}$, Pis'ma Zh. Eksp. Teor. Fiz. 24, 605 (1976) [JETP Lett. 24, 561 (1976)].

[21] M. C. Cross and W. F. Brinkman, Textural singularities in superfluid A-phase of ${ }^{3} \mathrm{He}$, J. Low Temp. Phys. 27, 683 (1977).

[22] M. M. Salomaa and G. E. Volovik, Half-Quantum Vortices in Superfluid ${ }^{3}$ He-A, Phys. Rev. Lett. 55, 1184 (1985).

[23] Chia-Ren Hu and K. Maki, Satellite magnetic resonances of a bound pair of half-quantum vortices in rotating superfluid ${ }^{3} \mathrm{He}-$ A, Phys. Rev. B 36, 6871 (1987).

[24] V. Vakaryuk and A. J. Leggett, Spin Polarization of HalfQuantum Vortex in Systems with Equal Spin Pairing, Phys. Rev. Lett. 103, 057003 (2009).

[25] D. Vollhardt and P. Wölfle, The Superfluid Phases of Helium 3 (Taylor and Francis, London, 1990).

[26] G. E. Volovik, Fermion zero modes on vortices in chiral superconductors, Pisma Zh. Eksp. Teor. Fiz. 70, 601 (1999) [JETP Lett. 70, 609 (1999)].

[27] N. Read and D. Green, Paired states of fermions in two dimensions with breaking of parity and time-reversal symmetries and the fractional quantum Hall effect, Phys. Rev. B 61, 10267 (2000).

[28] D. A. Ivanov, Non-Abelian Statistics of Half-Quantum Vortices in $p$-wave Superconductors, Phys. Rev. Lett. 86, 268 (2001).

[29] E. V. Thuneberg, Identification of Vortices in Superfluid ${ }^{3} \mathrm{He}-\mathrm{B}$, Phys. Rev. Lett. 56, 359 (1986).

[30] G. E. Volovik and M. M. Salomaa, Spontaneous breaking of axial symmetry in $v$-vortices in superfluid ${ }^{3} \mathrm{He}-\mathrm{B}$, Pis'ma Zh. Eksp. Teor. Fiz. 42, 421 (1985) [JETP Lett. 42, 521 (1985)].

[31] Y. Kondo, J. S. Korhonen, M. Krusius, V. V. Dmitriev, Yu. M. Mukharskiy, E. B. Sonin, and G. E. Volovik, Direct Observation of the Nonaxisymmetric Vortex in Superfluid ${ }^{3} \mathrm{He}-\mathrm{B}$, Phys. Rev. Lett. 67, 81 (1991).

[32] G. E. Volovik, Half quantum vortices in the B phase of superfluid ${ }^{3} \mathrm{He}$, Pis'ma Zh. Eksp. Teor. FIz. 52, 972 (1990) [JETP Lett. 52, 358 (1990)].

[33] M. A. Silaev, E. V. Thuneberg, and M. Fogelström, Lifshitz Transition in the Double-Core Vortex in ${ }^{3} \mathrm{He}-\mathrm{B}$, Phys. Rev. Lett. 115, 235301 (2015).

[34] V. P. Mineyev and G. E. Volovik, Planar and linear solitons in superfluid ${ }^{3} \mathrm{He}$, Phys. Rev. B 18, 3197 (1978).

[35] N. D. Mermin, The topological theory of defects in ordered media, Rev. Mod. Phys. 51, 591 (1979).

[36] L. Michel, Symmetry defects and broken symmetry. configurations hidden Symmetry, Rev. Mod. Phys. 52, 617 (1980).

[37] Y. Kondo, J. S. Korhonen, M. Krusius, V. V. Dmitriev, E. V. Thuneberg, and G. E. Volovik, Combined Spin-Mass Vortices with Soliton Tail in Superfluid ${ }^{3} \mathrm{He}-\mathrm{B}$, Phys. Rev. Lett. 68, 3331 (1992).

[38] S. Kang, S. W. Seo, H. Takeuchi, and Y. Shin, Observation of Wall-Vortex Composite Defects in a Spinor Bose-Einstein Condensate, Phys. Rev. Lett. 122, 095301 (2019).

[39] I-Kang Liu, Shih-Chuan Gou, and H. Takeuchi, Phase diagram of solitons in the polar phase of a Spin-1 Bose-Einstein condensate, arXiv:2002.6088.

[40] G. E. Volovik, Topological singularities on the surface of an ordered system, Pis'ma. Zh. Eksp. Teor. Fiz. 28, 65 (1978) [JETP Lett. 28, 59 (1978)].

[41] N. D. Mermin, E Pluribus Boojum: the physicist as neologist, Physics Today 34, 46 (1981).

[42] N. D. Mermin, V. P. Mineev, and G. E. Volovik, Topological analysis of cores of singularities in the ${ }^{3} \mathrm{He}-\mathrm{A}$, J. Low Temp. Phys. 33, 117 (1978).

[43] J. M. Cornwall, Center vortices, nexuses, and the GeorgiGlashow model, Phys. Rev. D 59, 125015 (1999).

[44] V. L. Golo and M. I. Monastyrsky, Gauge groups and topological invariants of vacuum manifolds, Ann. l'I. H. P. Phys. Theorique 28, 75 (1978).

[45] G. E. Volovik, Glass state of superfluid ${ }^{3} \mathrm{He}-\mathrm{A}$ in aerogel, Pis'ma Zh. Eksp. Teor. Fiz. 63, 281 (1996) [JETP Letters 63, 301 (1996)].

[46] V. V. Dmitriev, D. A. Krasnikhin, N. Mulders, A. A. Senin, G. E. Volovik, and A. N. Yudin, Orbital glass and spin glass states of ${ }^{3} \mathrm{He}-\mathrm{A}$ in aerogel, Pis'ma Zh. Eksp. Teor. Fiz. 91, 669 (2010) [JETP Lett. 91, 599 (2010)].

[47] G. E. Volovik, J. Rysti, J. T. Mäkinen, and V. B. Eltsov, Spin, orbital, Weyl and other glasses in topological superfluids, J. Low Temp. Phys. 196, 82 (2019).

[48] G. E. Volovik and V. P. Mineev, Investigation of singularities in superfluid ${ }^{3} \mathrm{He}$ and liquid crystals by homotopic topology methods, JETP Lett. 45, 1186 (1977).

[49] A. S. Schwarz, Field theories with no local conservation of the electric charge, Nucl. Phys. B 208, 141 (1982).

[50] M. M. Salomaa and G. E. Volovik, Cosmiclike domain walls in superfluid ${ }^{3} \mathrm{He}-\mathrm{B}$ : Instantons and diabolical points in $(\mathbf{k}, \mathbf{r})$ space, Phys. Rev. B 37, 9298 (1988).

[51] G. E. Volovik, Monopoles and fractional vortices in chiral superconductors, Proc. Natl. Acad. Sci. USA 97, 2431 (2000).

[52] S. Blaha, Quantization Rules for Point Singularities in Superfluid ${ }^{3} \mathrm{He}$ and Liquid Crystals, Phys. Rev. Lett. 36, 874 (1976). 
[53] G. E. Volovik and V. P. Mineev, Vortices with free ends in superfluid ${ }^{3} \mathrm{He}-\mathrm{A}$, Pis'ma Zh. Eksp. Teor. Fiz. 23, 647 (1976) [JETP Lett. 23, 593 (1976)].

[54] A. Saurabh and T. Vachaspati, Monopole-antimonopole: interaction, scattering and creation, Phil. Trans. R. Soc. A 377, 20190143 (2019).

[55] G. Lazarides and Q. Shafi, Monopoles, Strings, and Necklaces in $\mathrm{SO}(10)$ and $E_{6}$, J. High Energ. Phys. 10 (2019) 193.

[56] G. E. Volovik, Composite topological objects in topological superfluids, arXiv:1912.5962.

[57] N. Mermin and T.-L. Ho, Circulation and Angular Momentum in the A-Phase of Superfluid Helium-3, Phys. Rev. Lett. 36, 594 (1976).

[58] P. W. Anderson and G. Toulouse, Phase Slippage Without Vortex Cores: Vortex Textures in Superfluid ${ }^{3} \mathrm{He}$, Phys. Rev. Lett. 38, 508 (1977).

[59] V. R. Chechetkin, Types of vortex solutions in superfluid ${ }^{3} \mathrm{He}$, JETP Lett. 44, 766 (1976).

[60] G. E. Volovik and N. B. Kopnin, On the rotating ${ }^{3} \mathrm{He}-\mathrm{A}$, Pis'ma Zh. Eksp. Teor. Fiz. 25, 26 (1977) [JETP Lett. 25, 22 (1977)].

[61] H. K. Seppälä, P. J. Hakonen, M. Krusius, T. Ohmi, M. M. Salomaa, J. T. Simola, and G. E. Volovik, Continuous Vortices with Broken Symmetry in Rotating Superfluid ${ }^{3} \mathrm{He}-\mathrm{A}$, Phys. Rev. Lett. 52, 1802 (1984).

[62] J. P. Pekola, K. Torizuka, A. J. Manninen, J. M. Kyynäräinen, and G. E. Volovik, Observation of a Topological Transition in the ${ }^{3}$ He-A Vortices, Phys. Rev. Lett 65, 3293 (1990).
[63] T. D. C. Bevan, A. J. Manninen, J. B. Cook, J. R. Hook, H. E. Hall, T. Vachaspati, and G. E. Volovik, Momentum creation by vortices in superfluid ${ }^{3} \mathrm{He}$ as a model of primordial baryogenesis, Nature (London) 386, 689-692 (1997).

[64] S. M. Dahir, A. F. Volkov, and I. M. Eremin, Interaction of Skyrmions and Pearl Vortices in Superconductor-Chiral Ferromagnet Heterostructures, Phys. Rev. Lett. 122, 097001 (2019).

[65] S. Rex, I. V. Gornyi, and A. D. Mirlin, Majorana bound states in magnetic skyrmions imposed onto a superconductor, Phys. Rev. B 100, 064504 (2019).

[66] A. B. Vorontsov and J. A. Sauls, Crystalline Order in Superfluid ${ }^{3}$ He Films, Phys. Rev. Lett. 98, 045301 (2007).

[67] L. V. Levitin, B. Yager, L. Sumner, B. Cowan, A. J. Casey, J. Saunders, N. Zhelev, R. G. Bennett, and J. M. Parpia, Evidence for a Spatially Modulated Superfluid Phase of ${ }^{3} \mathrm{He}$ Under Confinement, Phys. Rev. Lett. 122, 085301 (2019).

[68] J. Shook, V. Vadakumbatt, P. Senarath Yapa, C. Doolin, R. Boyack, P. H. Kim, G. G. Popowich, F. Souris, H. Christani, J. Maciejko, and J. P. Davis, Stabilized Pair Density Wave via Nanoscale Confinement of Superfluid ${ }^{3} \mathrm{He}-\mathrm{A}$, Phys. Rev. Lett. 124, 015301 (2020).

[69] C. Nash and S. Sen, Topology and Geometry for Physicists (Academic Press, Cambridge, 1988).

[70] T. H. R. Skyrme, A unified field theory of mesons and baryons, Nucl. Phys 31, 556 (1962).

[71] U. A. Khawaja and H. Stoof, Skyrmions in a ferromagnetic Bose-Einstein Condensate, Nature (London) 411, 918 (2001). 